\title{
Plant resource uses and losses in a small maroon community in a Colombian Pacific hot spot
}

\author{
DANIELLY VASCONCELOS TRAVASSOS DE LIMA ${ }^{1}$, LAURA LOZADA ORDÓÑEZ ${ }^{2}$, \\ DENISE DIAS DA CRUZ ${ }^{3, \vartheta}$ \\ 1Programa de Pós-Graduação em Desenvolvimento e Meio Ambiente, PRODEMA, Universidade Federal da Paraíba. João Pessoa, PB, 58051-900, Brazil \\ ${ }^{2}$ Representante da Fundação Pacifilia e consultor em Desenvolvimento Comunitário e Meio Ambiente. Bogotá, Colombia \\ ${ }^{3}$ Departamento de Sistemática e Ecologia, Centro de Ciências Exatas e da Natureza, Universidade Federal da Paraíba, João Pessoa, PB, 58051-900, \\ Brazil. `email: denidcruz@dse.ufpb.br
}

Manuscript received: 26 March 2021. Revision accepted: 4 May 2021

\begin{abstract}
Lima DVT, Ordónez LL, da Cruz DD. 2021. Plant resource uses and losses in a small maroon community in a Colombian Pacific hot spot. Biodiversitas 22: 3031-3041. The Colombian Pacific is a world biodiversity hotspot, and traditional communities of the region have high cultural diversity, such as the Jovi maroon community, which shows economic and cultural practices rely on regional ecosystem services. This study aimed to conduct an ethnobotanical survey in the Jovi community, specifically focused on the identification of the plants used, the purpose of use, losses in the use of species, the conservation status of the species, and if there are differences regarding plant use knowledge between genres. Participant observation was conducted associated with interviews for data collection with 26 local experts. The Jovi community uses a wide variety of plants (135 species belonging to 57 families). Cultivated species showed the highest use values in the community. The most important uses were medicinal, food, and technological, respectively. Use losses were verified, mainly, for the species used as food. Two species deserve attention, i.e. Minquartia guianensis (Near Threatened - IUCN), and Pelliciera rhizophorae (Vulnerable - IUCN), both obtained by extractivism and have timber use. There was no difference in knowledge between men and women. Cultural changes may be delimiting changes in the use of plant species in the community.
\end{abstract}

Keywords: Ethnobotany, knowledge and gender, medicinal plants, traditional populations

\section{INTRODUCTION}

Plant resources are important ecosystem services for the survival of traditional populations, provide supplies, and, in many cases, they are bare for the maintenance of culture and economy (MEA 2005; Diegues 2019). The local biodiversity is directly related to the guarantee of the quality of life among these populations with limited financial resources, who rely on nature to extract food, medicines, fuel, and building materials (Maroyi 2017).

Traditional populations are known for their knowledge of the management of natural resources, extrapolating the purpose of survival and reaching wider scopes, such as culture and economy (Kakoty 2018). Therefore, the propagation of this knowledge related to the sustainable use of plant species should be stimulated in preservation management and environmental conservation studies aiming at a positive symbiotic relationship between society and nature (Harisha et al. 2016; Diegues 2019).

These man-nature interactions gain more prominence, nowadays, when located in areas of high biodiversity. In areas featuring exceptional concentrations of endemic species, considered hotspots (Myers 2000), it is essential to understand the degree of threat to which biodiversity is subject, in addition to its importance to cultural and social relations (Myers 2000). The Colombian Pacific is one of these world regions with remarkable biodiversity, sheltering several populations with traditional practices
(Mittermeier et al. 2004). In this context, ethnobotanical studies can help to understand this relationship between the local community and the use of plant resources in the area, aiming to promote environmental maintenance (Myers 2000; Restrepo 2013).

The Afro-descendant community of Jovi is located in the northern Pacific region of Colombia, and its cultural and economic practices are based on regional ecosystem services. Agriculture and ecotourism are important financial support activities for the inhabitants who still resist migrating to the urban zone, preserving their traditional practices (Ordóñez et al. 2018). There is a clear specification of female activities in the Jovi community, such as the community bank management, the cultivation of home-grown vegetable gardens, mystical religious rituals, food preparation, and the propagation of traditional culinary recipes (Ordõnez et al. 2018).

In this scenario, it is worth noting that, despite possessing traditional knowledge, the natives have a perception of loss of species and ecosystem services and even recognize possible justifications for the inappropriate use of natural resources, such as inadequate fishing practices and negligence with the mangroves, which has reduced its physical dimension in the last few years (Restrepo 2013; Ordõnez et al. 2018).

In this context, this study aims to conduct an ethnobotanical survey in the Jovi community, specifically focusing on a) the identification of the plants used by the 
community and their purpose of use; b) if the community identify losses in the use of any species; c) if there are differences between men and women regarding the use of plant resources; and d) the ecological threatened species used by the community. The hypotheses of this study are: there is a significant loss in the use of some plant species, and there is a difference between genders regarding the knowledge of the different categories of plant use.

\section{MATERIALS AND METHODS}

\section{Study area}

The study was conducted at the Jovi community, in the Gulf of Tribugá, located in the municipality of Nuqui, Department of Chocó, in the Pacific Colombian region. The Jovi community possesses an area of 37.48 hectares (Ordõnez et al. 2018) and is located in one of the richest biomes in endemic species in the world (Myers et al. 2000).

The Department of Chocó is an area of high biological diversity, with the highest abundance of mammals in the Pacific region, besides presenting one of the highest rainfall rates in the world (approximately 9,382 $\mathrm{mm} /$ year), generating a wide water network (Braham and Blandón 2013). The region is characterized by the presence of rainforests, with temperatures ranging from 22 to $26^{\circ} \mathrm{C}$, without distinct drought periods, favoring biomass development and the presence of forest landscapes, cultivation areas, rivers, mangroves, beaches, streams, and creeks (Zuluaga and Ramírez 2015).

The Jovi community has about 170 inhabitants living in 67 homes, most of whom are of African descent, who populated the village more than 70 years ago. Agriculture is the main economic activity due to the abundance of natural resources in the place, but with the emergence of tourism, beginning in the 1980 s, this activity currently represents $37 \%$ of the main income of the community (Ordõnez et al. 2018). The Jovi community inhabitants rely on a discontinuous power supply, untreated water sources, and the absence of sewage and waste collection (Ordõnez et al. 2018).

The inhabitants have a perception that they live in a harmonious relationship with the life cycles of nature, which has been recorded in the ethnic-development plan and in some studies in which the population was the object of study (Riscales 2005).

\section{Ethnobotanical data collection}

The study had exploratory, qualitative-quantitative approaches. The data were collected from December 2015 to April 2016, and from April to July 2020. Participant observation and semi-structured interviews were employed. The interviews were aimed to gather data on the plant species used, their uses and collection, the plant parts used, and whether their uses were current or potential to record the loss of species.

The interviews were conducted with experts defined through the "snowball" technique, which is a useful network sampling method to study populations hard to be accessed or studied (Bernard 2017). In this manner, there were 26 experts in total in the fields of fishing, agriculture, and tourism, which are the three main economic activities of the community (Ordõnez et al. 2018). The sample showed a heterogenous formation regarding age (54\% from 27 to 59 years, and $46 \%$ were 60 years or older), and gender $(62 \%$ of the interviewees were men and $38 \%$ were women), and most were of local origin ( $85 \%$ local, $15 \%$ non-local).

The plants indicated by the interviewees were collected through the guided tour technique (Albuquerque et al. 2014), and the non-collected species were identified through pictures by different people in the community who used them. The plants were deposited in the Herbarium of the Technological University of Choco (UTCH). The identifications followed the APG IV classification, and the scientific names were confirmed according to the International Plant Names Index (https://www.ipni.org/), Universidad Nacional de Colombia databases (http://www.biovirtual.unal.edu.co/nombrescomunes/es/), and the Flora do Brasil http://floradobrasil.jbrj.gov.br/reflora). The conservation status was checked in the IUCN Red List of Threatened Species (https://www.iucnredlist.org/).

\section{Ethical issues}

Although the study was developed in a Colombian community, since the researchers are affiliated with a Brazilian institution, the Brazilian legislation rules were followed regarding the ethical issues of the study. In Colombia, there is no requirement for research approved by an ethics committee. Nevertheless, each participant was informed on the purpose of the study, and, if in agreement, signed the Informed Consent Form (TCLE) required by the National Health Council through the Research Ethics Committee (Resolution 196/96).

The present study was approved by the Human Research Ethics Committee (CEP) of the Center of Health Sciences (CCS) of the Federal University of Paraíba (UFPB) under the protocol number CAAE 56813716.4.0000.5188 and received the approval of the local ethnic authority, the General Community Council Los Riscales.

\section{Data analysis}

The plants were classified as eight use-categories for data analysis, i.e. medicinal, food, technological, building materials, fuel, mystic/religious, and others (ornamental, tourism, and recreation) (Galeano 2000). For each species, the Total Use Value was calculated through the formula adapted from Rossato et al. (1999): UV $=\Sigma U / n$, where UV: use-value of the species, U: number of total quotations per species, and n: number of informants. The Current Use Value and the Potential Use Value were also calculated following Lucena et al. (2012), according to the formulas, respectively: $U V a=\Sigma U$ current $/ n$, where UVa: current usevalue of the species, $U$ : number of uses of the species mentioned by the informant, and $n$ : number of informants; and $U V p=\Sigma U$ potential $/ \mathrm{n}$, where $U V p$ : potential use-value of the species, $U$ : number of potential uses of the species mentioned by the informant, and $\mathrm{n}$ : number of informants. This differentiation in the potential use value aims to 
demonstrate species with loss of current use. The Wilcoxon test was performed to compare the knowledge between men and women. This test was used since the data did not have a normal distribution. The test was performed in the $\mathrm{R}$ Studio (RStudio Team 2020).

\section{RESULTS AND DISCUSSION}

The Jovi community used 135 plant species belonging to 57 families. The families of Lamiaceae and Poaceae showed the highest number of species, respectively. Plants were obtained by extractivism (70 species) or were cultivated (59 species), and 6 species were obtained in both ways (Table 1).

Almost $1 / 3$ of the species $(34,07 \%)$ are listed in the IUCN Red List: 42 species are classified as Least Concern, three species are Data Deficient, one species is classified as Near Threatened, and one species is classified as Vulnerable (Table 1). The species classified in the most endangers categories were both obtained by extractivism (Minquartia guianensis and Pelliciera rhizophorae), and have timber use (Table 1).

Plant uses were grouped into seven categories and 49 species were indicated with more than one use category, demonstrating the versatility of use (Table 1). The medicinal category represented the highest number of species informed by the experts and fuel received fewer citations (Figure 1).

Most species (106) showed low total use-values, ranging from 0.0 to 0.2 . Just two species showed a total use value higher than 0.81 , i.e. Oryza sativa and Musa $x$ paradisiaca (Figure 2).

The ranking of the species considered most important by the community highlighted 10 species, which can be organized in the five most important positions (Table 2) (after this position, UV is very similar to the other species). Four species were obtained by extractivism, five were cultivated, and one species was obtained by both forms. Oryza sativa (arroz) was the most important species

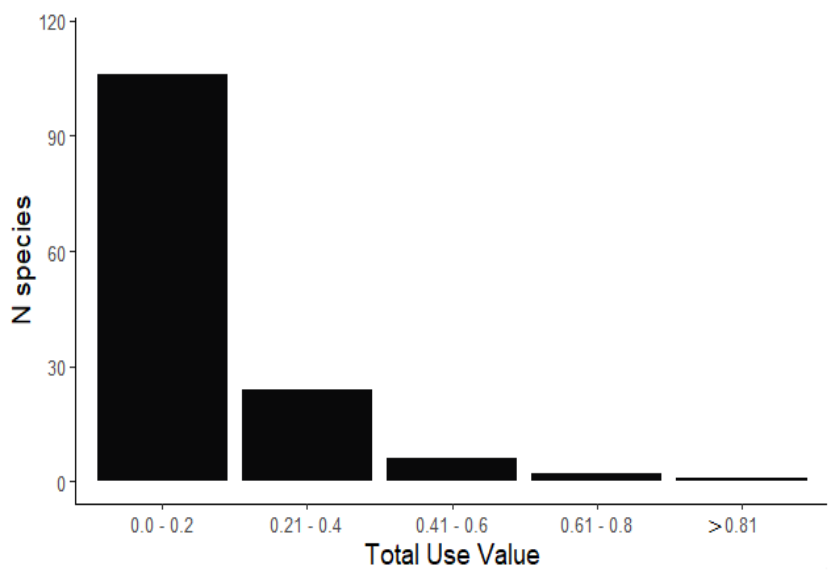

Figure 2. Class distribution of the use-value of plant species cited by local experts in the Jovi community, Colombian Pacific region identified by the community considering current, potential, and total UV (Table 2). The potential use was cited to the varieties 'fortune' and 'uringa' that are not currently planted. Musa x paradisiaca (plátano) was the second most important species recognized as potential UV. Oryza sativa and Musa x paradisiaca are both used for food purposes and are frequently present in the meals of the community. The third most important species in the total UV and second most important in the potential UV was Dipteryx odorata, which was obtained just by extractivism and is classified as Data Deficient in the IUCN Red List (Tables 1, 2). We also highlight two species: Gliricidia sepium (fifth-highest current UV) and Nectandra acutifolia (fifthhighest potential VU), suggesting the use of these species have decreased in the last years (Table 2). Both species are classified as Least Concern on the IUCN Red List (Table 1).

The interviews were conducted with 16 men and 10 women considered experts within the Jovi community. The results showed no difference in knowledge between men and women $(\mathrm{W}=14646 ; \mathrm{p}=0.3006)$ (Figure 3$)$.

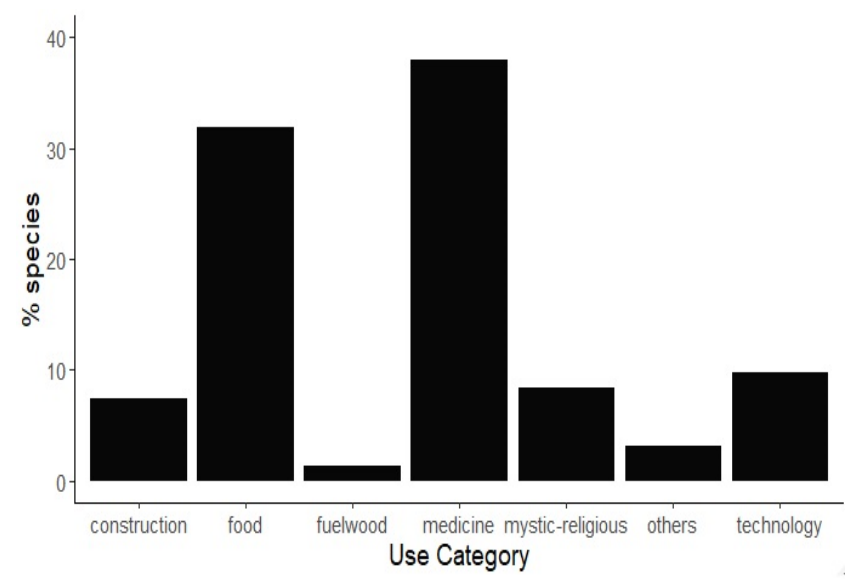

Figure 1. Distribution of species according to the use categories cited by local experts in the Jovi community, Colombian Pacific

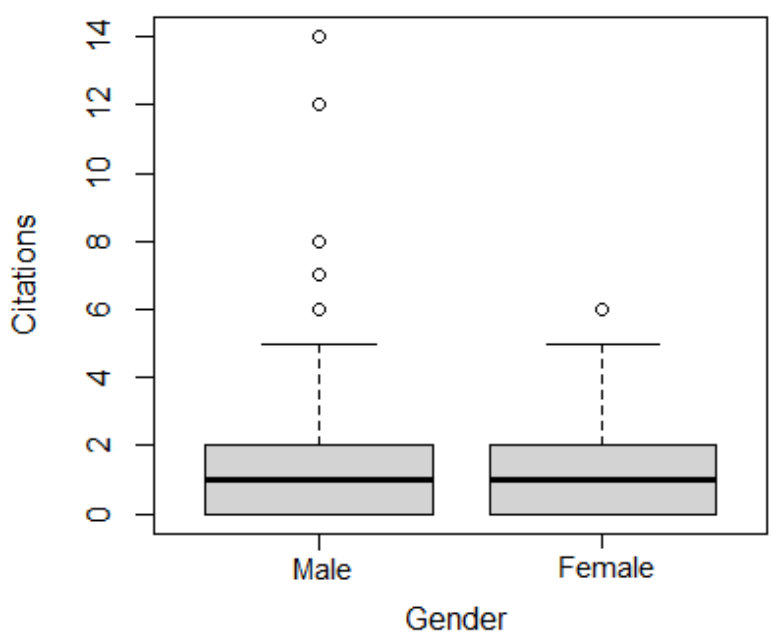

Figure 3. Ethnobotanical knowledge of men and women in the Jovi community, Colombian Pacific 
Table 1. Classification of the plants cited by local experts of the Jovi community, Colombian Pacific.

\begin{tabular}{|c|c|c|c|c|c|c|c|}
\hline Family & Species & Popular name & $\mathbf{U V} \mathbf{a}$ & $\mathbf{U V} \mathbf{V}_{\mathbf{p}}$ & $\mathbf{U V}_{\mathbf{t}}$ & $\mathbf{U C}$ & P or $\mathbf{E}$ \\
\hline \multicolumn{8}{|c|}{ Acanthaceae } \\
\hline & $\begin{array}{l}\text { (LC) Trichanthera gigantea Humb. \& } \\
\text { Bonpl. Ex. Steud. }\end{array}$ & Nascedera & 0.12 & 0.00 & 0.12 & me & $P$ \\
\hline & Justicia sp. & Riñonera & 0.04 & 0.00 & 0.04 & me & $P$ \\
\hline \multicolumn{8}{|c|}{ Amaranthaceae } \\
\hline & Alternanthera lanceolata Schinz & Descansé & 0.04 & 0.00 & 0.04 & me & $\mathrm{P}$ \\
\hline & Chenopódium ambrosioides Bert ex. Steud & Paico & 0.23 & 0.00 & 0.23 & me & $\mathrm{P}$ \\
\hline \multicolumn{8}{|c|}{ Amaryllidaceae } \\
\hline & Allium sativum $\mathrm{L}$. & Ajo & 0.08 & 0.04 & 0.12 & fo, me & $\mathrm{P}$ \\
\hline & Allium cepa $\mathrm{L}$. & Cebolla, cebolla de rama & 0.31 & 0.04 & 0.35 & fo, me & $\mathrm{P}$ \\
\hline \multicolumn{8}{|c|}{ Anacardiaceae } \\
\hline & Anacardium excelsum Skeeks & Espavé & 0.27 & 0.15 & 0.42 & tc & $\mathrm{E}$ \\
\hline & (LC) Spondias mombin Jacq. & Ovo & 0.12 & 0.00 & 0.12 & fo, me & $\mathrm{E} / \mathrm{P}$ \\
\hline \multicolumn{8}{|c|}{ Annonaceae } \\
\hline & (LC) Annona muricata $\mathrm{L}$. & Guanábana & 0.04 & 0.00 & 0.04 & me & $\mathrm{P}$ \\
\hline & (LC) Rollinia danforthii Standl. & Gúasimo blanco & 0.35 & 0.00 & 0.35 & me & $\mathrm{E}$ \\
\hline \multicolumn{8}{|c|}{ Apiaceae } \\
\hline & Eryngium foetidum $\mathrm{L}$. & Cilantro & 0.08 & 0.00 & 0.08 & fo & $\mathrm{P}$ \\
\hline \multicolumn{8}{|l|}{ Araceae } \\
\hline & Monstera adansonii Schott & costilla de Adán & 0.35 & 0.00 & 0.35 & $\mathrm{me}, \mathrm{m} / \mathrm{r}$ & $\mathrm{E}$ \\
\hline & Xanthosoma sagittifolium Liebm. & Otó & 0.04 & 0.00 & 0.04 & fo & $\mathrm{P}$ \\
\hline & Colocasia esculenta (L.) Schott & Papachina & 0.04 & 0.00 & 0.04 & fo & $P$ \\
\hline \multicolumn{8}{|c|}{ Arecaceae } \\
\hline & (LC) Iriartea deltoidea Ruiz \& Pav. & Chonta & 0.04 & 0.00 & 0.04 & $\mathrm{tc}$ & $\mathrm{E}$ \\
\hline & Bactris gasipaes Kunth & Chontaduro & 0.27 & 0.23 & 0.50 & fo & $\mathrm{P}$ \\
\hline & Astrocaryum standleyanum L.H.Bailley & Chunga & 0.08 & 0.00 & 0.08 & tc & $\mathrm{E}$ \\
\hline & Cocos nucifera $\mathrm{L}$ & Coco & 0.54 & 0.04 & 0.58 & tc, fo, me & $\mathrm{E} / \mathrm{P}$ \\
\hline & Oenocarpus bataua Mart. & Mil pesos, trupa & 0.08 & 0.04 & 0.12 & fo & $\mathrm{E}$ \\
\hline & Syagrus sancona H.Karst. & Palma zancona & 0.04 & 0.00 & 0.04 & tc & $\mathrm{E}$ \\
\hline & Phytelephas macrocarpa Ruiz \& Pav. & Tagua & 0.08 & 0.00 & 0.08 & tc & $\mathrm{E}$ \\
\hline \multicolumn{8}{|c|}{ Aristolochiaceae } \\
\hline \multirow{2}{*}{\multicolumn{8}{|c|}{ 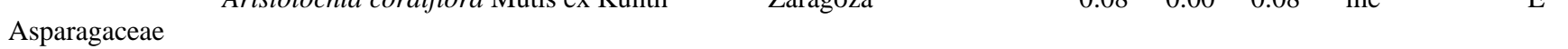 }} \\
\hline & & & & & & & \\
\hline \multicolumn{8}{|c|}{ Cordyline fruticosa Göpp. } \\
\hline & Pseudelephantopus sp. & Consuelda, chicoria & 0.15 & 0.00 & 0.15 & me, out & $P$ \\
\hline & Adenostemma platyphyllum Cass. & Doñajuana & 0.12 & 0.00 & 0.12 & me & $\mathrm{P}$ \\
\hline & Achillea millefolium $\mathrm{L}$. & Hierba de carpintero & 0.15 & 0.00 & 0.15 & fo, me & $\mathrm{E} / \mathrm{P}$ \\
\hline & Ageratum conyzoides Sieber ex. Steud. & Hierba de chiva & 0.08 & 0.00 & 0.08 & me & $\mathrm{E}$ \\
\hline & Neurolaena lobata $\mathrm{R} . \mathrm{Br}$. & Venadillo & 0.08 & 0.00 & 0.08 & me & $\mathrm{E}$ \\
\hline & Ambrosia cumanensis Kunth & Altamisa & 0.04 & 0.00 & 0.08 & $\mathrm{~m} / \mathrm{r}$ & $\mathrm{P}$ \\
\hline & Artemisia sodiroi Hieron. & Alhucema & 0.15 & 0.08 & 0.23 & me & $\mathrm{P}$ \\
\hline & Sphagneticola trilobata (L.) Pruski & Botoncillo & 0.08 & 0.00 & 0.08 & me & $\mathrm{E}$ \\
\hline \multicolumn{6}{|c|}{ 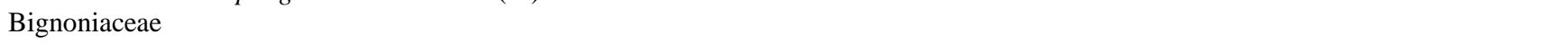 } & & \\
\hline & (LC) Crescentia cujete Vell. & Totúmo & 0.04 & 0.00 & $\begin{array}{l}0.08 \\
0.04\end{array}$ & $\begin{array}{l}\text { lu, } 10 \\
\text { tc }\end{array}$ & $\begin{array}{l}\mathrm{E} \\
\mathrm{E}\end{array}$ \\
\hline & (LC) Jacaranda hesperia Dugand & Tunisco & 0.12 & 0.00 & 0.12 & me & $\mathrm{E}$ \\
\hline \multicolumn{8}{|c|}{ Boraginaceae } \\
\hline \multicolumn{8}{|c|}{ (LC) Varronia spinescens (L.) Borhidi } \\
\hline & Ananas comosus (L.) Merr. & Piña & 0.08 & 0.00 & 0.08 & fo & $\mathrm{P}$ \\
\hline & (LC) Tillandsia usneoides (L.) L. & Salvajina, gallito & 0.19 & 0.00 & 0.19 & $\mathrm{~m} / \mathrm{r}$ & $\mathrm{E}$ \\
\hline \multicolumn{8}{|c|}{ Burseraceae } \\
\hline \multicolumn{8}{|c|}{ Protium amplum Cuatrec. } \\
\hline \multirow{2}{*}{\multicolumn{8}{|c|}{ Mora oleífera Ducke }} \\
\hline & & & & & & & \\
\hline & Carica papaya $\mathrm{L}$. & Papaya & 0.08 & 0.00 & 0.08 & fo & $\mathrm{P}$ \\
\hline Chloran & & & & & & & \\
\hline$C$ & (LC) Hedyosmum scaberrimum Standl. & Rodilla de pollo, rodillon & 0.04 & 0.00 & 0.04 & me & $\mathrm{E}$ \\
\hline & (LC) Licania fuchsii Prance & Carbonero & 0.04 & 0.00 & 0.04 & $\mathrm{fu}$ & E \\
\hline
\end{tabular}




\begin{tabular}{|c|c|c|c|c|c|c|c|}
\hline \multicolumn{8}{|l|}{ Convolvulaceae } \\
\hline & (DD) Ipomoea batatas (L). Lam. & Batata & 0.04 & 0.00 & 0.04 & $\mathrm{~m} / \mathrm{r}$ & $\mathrm{P}$ \\
\hline & Tripogandra serrulata (Vahl) Handlos & Siempre viva & 0.08 & 0.00 & 0.08 & me & $\mathrm{E}$ \\
\hline & Bryophyllum pinnatum Kurz & Hierba santa & 0.08 & 0.04 & 0.12 & me & $\mathrm{E} / \mathrm{P}$ \\
\hline & (LC) Carludovica palmata Ruiz \& Pav. & Iraca & 0.15 & 0.00 & 0.15 & tc, fo & $\mathrm{E}$ \\
\hline Cyperaceae & (LC) Kyllinga odorata Liebm. & Espadilla & 0.08 & 0.04 & 0.12 & tc, me & $\mathrm{E}$ \\
\hline Dioscoreaceae & Dioscorea trifida L.f. & Name & 0.08 & 0.00 & 0.08 & fo & $\mathrm{P}$ \\
\hline & Euphorbiaceae & Yuca & 0.31 & 0.00 & 0.31 & fo & $\mathrm{P}$ \\
\hline Fabaceae & $\begin{array}{l}\text { (LC) Andira inermis (W.Wright) Kunth ex } \\
\text { DC. }\end{array}$ & Amargo & 0.12 & 0.03 & 0.15 & $\mathrm{cs}, \mathrm{tc}, \mathrm{me}$ & $\mathrm{E}$ \\
\hline & $\begin{array}{l}\text { (DD) Dipteryx odorata (Aubl.) Willd. } \\
\text { (LC) Senna reticulata (Willd.) H.S.Irwin \& } \\
\text { Barneby }\end{array}$ & $\begin{array}{l}\text { Choibá } \\
\text { Galve }\end{array}$ & $\begin{array}{l}0.46 \\
0.08\end{array}$ & $\begin{array}{l}0.23 \\
0.00\end{array}$ & $\begin{array}{l}0.69 \\
0.08\end{array}$ & $\begin{array}{l}\text { cs, tc } \\
\text { me }\end{array}$ & $\begin{array}{l}E \\
E\end{array}$ \\
\hline & Lens culinaris Medik. & Lenteja & 0.08 & 0.00 & 0.08 & fo, me & $P$ \\
\hline & (LC) Gliricidia sepium Kunth & Matarratón & 0.46 & 0.00 & 0.46 & $\mathrm{fu}, \mathrm{me}$ & $\mathrm{P}$ \\
\hline & $\begin{array}{l}\text { (LC) Zygia longifolia (Humb. \& Bonpl. Ex } \\
\text { Willd.) Britton \& Rose }\end{array}$ & Pichinde & 0.04 & 0.00 & 0.04 & out & $\mathrm{E}$ \\
\hline & Senna occidentalis (L.) Link & Potra & 0.15 & 0.00 & 0.15 & me & $P$ \\
\hline Fitolaceas & Phytolacca bogotensis Kunth & Guaba & 0.04 & 0.00 & 0.04 & me & $\mathrm{E}$ \\
\hline & Drymonia killipii Wiehler & Desbaratadora & 0.04 & 0.00 & 0.04 & me & E \\
\hline & Saintpaulia ionantha H.Wendl. & Violeta & 0.04 & 0.00 & 0.04 & me & $\mathrm{P}$ \\
\hline Heliconaceae & Heliconia psittacorum L.f. & Heliconia & 0.12 & 0.00 & 0.12 & out, me & E \\
\hline \multicolumn{4}{|l|}{ Humiriaceae } & & & cs & $\mathrm{P}$ \\
\hline \multicolumn{8}{|l|}{ Lamiaceae } \\
\hline & Scutellaria pseudocoleus Fern.Alonso & Albacón & 0.08 & 0.00 & 0.08 & fo, me & $\mathrm{P}$ \\
\hline & Ocimum campechianum Chapm. & Albahaca & 0.15 & 0.00 & 0.15 & fo, $\mathrm{m} / \mathrm{r}$ & $P$ \\
\hline & Lepechinia sp. & Albahaca blanca & 0.08 & 0.00 & 0.08 & me & $P$ \\
\hline & Ocimum sp. & Albahaca morada & 0.04 & 0.00 & 0.04 & fo & $P$ \\
\hline & Hyptis verticillata Jacq. & Gallinaza & 0.12 & 0.03 & 0.15 & me & $\mathrm{E}$ \\
\hline & Origanum vulgare L. & Orégano & 0.12 & 0.00 & 0.12 & fo, me & $\mathrm{P}$ \\
\hline & Clinopodium brownei (Sw.) Kuntze & Poleo & 0.23 & 0.00 & 0.23 & tc, fo, me & $P$ \\
\hline & Rosmarinus officinalis $\mathrm{L}$. & Romero & 0.12 & 0.00 & 0.12 & fo, me & $\mathrm{P}$ \\
\hline & Tectona grandis L.f. & Teka & 0.08 & 0.0 & 0.08 & $\mathrm{cs}$ & $P$ \\
\hline & Hyptis sp. & Toronjil & 0.04 & 0.0 & 0.04 & me & $P$ \\
\hline \multicolumn{8}{|l|}{ Lauraceae } \\
\hline & Pleurothyrium glabritepalum van der Werff & Perena & 0.38 & 0.00 & 0.38 & cs, tc, out & $\mathrm{E}$ \\
\hline \multicolumn{7}{|c|}{ E } & $P$ \\
\hline & (LC) Ochroma pyramidale (Cav. Ex Lam.) Urb. & Balso & 0.35 & 0.03 & 0.38 & tc, me, out & $\mathrm{E}$ \\
\hline & Theobroma cacao L. & Chocolate, Cacao & 0.12 & 0.03 & 0.15 & fo & $\mathrm{P}$ \\
\hline & Sida rhombifolia $\mathrm{L}$. & Escobabosa & 0.12 & 0.00 & 0.12 & me & $\mathrm{E} / \mathrm{P}$ \\
\hline & (LC) Luehea seemannii Planch. \& Triana & Guásimo colorado & 0.23 & 0.00 & 0.23 & la, tc, me & $\mathrm{E}$ \\
\hline & Talipariti tiliaceum (L.) Fryxell & Majaguo & 0.04 & 0.00 & 0.04 & me & E \\
\hline & Malachra alceifolia Jacq. & Malva & 0.12 & 0.00 & 0.12 & me & $\mathrm{P}$ \\
\hline & Hibiscus rosa-sinensis $\mathrm{L}$. & Papo, resucito, san joaquin & 0.31 & 0.00 & 0.31 & $\mathrm{me}, \mathrm{m} / \mathrm{r}$ & $\mathrm{E}$ \\
\hline & (LC) Apeiba membranacea Spruce ex Benth. & Peine de mono & 0.08 & 0.00 & 0.08 & cs & $\mathrm{E}$ \\
\hline \multicolumn{8}{|c|}{ Melastomataceae } \\
\hline & Nepsera aquática Naudin & Cana agria & 0.19 & 0.00 & 0.19 & tc, fo, me & $\mathrm{E} / \mathrm{P}$ \\
\hline \multirow{2}{*}{ Meliaceae } & (LC) Clidemia septuplinervia Cogn. & Mora & 0.27 & 0.00 & 0.27 & $\mathrm{cs}, \mathrm{tc}$, fo, me & $\mathrm{E}$ \\
\hline & (LC) Carapa guianensis Aubl. & Gûina & 0.15 & 0.00 & 0.15 & tc & E \\
\hline \multirow{2}{*}{ Moraceae } & (LC) Poulsenia armata (Miq.) Standl. & Damagua & 0.04 & 0.00 & 0.04 & tc & $\mathrm{E}$ \\
\hline & (LC) Ficus maxima Mill. & Higuéron & 0.15 & 0.04 & 0.19 & tc, me & $\mathrm{E}$ \\
\hline
\end{tabular}




\begin{tabular}{|c|c|c|c|c|c|c|c|}
\hline & $\begin{array}{l}\text { (LC) Batocarpus costaricensis Standl. \& } \\
\text { L.O.Williams }\end{array}$ & Mare & 0.08 & 0.00 & 0.08 & fo & $\mathrm{E}$ \\
\hline & Castilla tипи Hemsl. & Caucho & 0.04 & 0.00 & 0.04 & fo & $\mathrm{E}$ \\
\hline & Artocarpus altilis (Parkinson) Fosberg & Pepadepán & 0.12 & 0.00 & 0.12 & fo, out & $\mathrm{P}$ \\
\hline Vilusaceac & Musa acuminata Colla & Banano & 0.23 & 0.00 & 0.23 & fo & $\mathrm{P}$ \\
\hline & Musa x paradisiaca $\mathrm{L}$. & Colino, Felipita, Plátano & 0.81 & 0.00 & 0.81 & fo & $\mathrm{P}$ \\
\hline & Musa sp. & Guineo pajarito & 0.04 & 0.00 & 0.04 & me & $\mathrm{P}$ \\
\hline & Musa acuminata cv. 'Sucrier' & Primitivo & 0.04 & 0.00 & 0.04 & fo & $\mathrm{P}$ \\
\hline & Musa sp. & Tres esquinas & 0.08 & 0.00 & 0.08 & $\mathrm{me}, \mathrm{m} / \mathrm{r}$ & $\mathrm{E}$ \\
\hline \multicolumn{8}{|l|}{ Myrtaceae } \\
\hline & (LC) Psidium guajava $\mathbf{L}$. & $\begin{array}{l}\text { Arrayan } \\
\text { Guayabo }\end{array}$ & $\begin{array}{l}0.04 \\
0.23\end{array}$ & $\begin{array}{l}0.00 \\
0.00\end{array}$ & $\begin{array}{l}0.04 \\
0.23\end{array}$ & $\begin{array}{l}\text { me } \\
\text { fo, me }\end{array}$ & $\begin{array}{l}\mathrm{P} \\
\mathrm{P}\end{array}$ \\
\hline Olacaceae & & Guayacán, Guayacán negro & 0.23 & 0.15 & 0.38 & cs & E \\
\hline Oxalidaceae & Averrhoa carambola $\mathrm{L}$. & Carambolo & 0.04 & 0.00 & 0.04 & fo & $\mathrm{P}$ \\
\hline 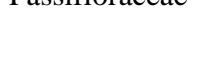 & Passiflora vitifolia Kunth & Granadillo & 0.15 & 0.12 & 0.27 & cs & $\begin{array}{l}\text { E } \\
\mathrm{E}\end{array}$ \\
\hline Pellicieraceae & $\begin{array}{l}\text { (VU) Pelliciera rhizophorae Planch. \& } \\
\text { Triana }\end{array}$ & Piñuelo & 0.04 & 0.00 & 0.04 & tc & $\mathrm{E}$ \\
\hline Phytolaccaceae & Petiveria $\mathrm{L}$. & Anamú & 0.23 & 0.00 & 0.23 & $\mathrm{me}, \mathrm{m} / \mathrm{r}$ & $\mathrm{P}$ \\
\hline pe & $\begin{array}{l}\text { Piper } \mathrm{sp} 1 . \\
\text { Piper } \mathrm{sp} 2 . \\
\text { Peperomia } \mathrm{sp} . \\
\text { Piper peltatum } \mathrm{L} . \\
\text { (LC) Piper auritum Kunth }\end{array}$ & $\begin{array}{l}\text { Celedonia } \\
\text { Coronillo } \\
\text { Hierba de hormiga } \\
\text { Santa maria boba } \\
\text { Santa maria de aniz }\end{array}$ & $\begin{array}{l}0.04 \\
0.35 \\
0.04 \\
0.15 \\
0.27\end{array}$ & $\begin{array}{l}0.00 \\
0.00 \\
0.00 \\
0.00 \\
0.00\end{array}$ & $\begin{array}{l}0.04 \\
0.35 \\
0.04 \\
0.15 \\
0.27\end{array}$ & $\begin{array}{l}\mathrm{me} \\
\mathrm{me}, \mathrm{m} / \mathrm{r} \\
\mathrm{m} / \mathrm{r} \\
\mathrm{me} \\
\text { fo, me }\end{array}$ & $\begin{array}{l}P \\
E \\
E \\
E \\
E\end{array}$ \\
\hline Plantaginaceae & $\begin{array}{l}\text { (LC) Plantago major } \mathbf{L} \text {. } \\
\text { Scoparia dulcis } \mathrm{L} \text {. } \\
\text { Conobea scoparioides Benth. } \\
\text { (LC) Plantago major } \mathbf{L} .\end{array}$ & $\begin{array}{l}\text { Amaranto } \\
\text { Escubilla } \\
\text { Hierba de sapo } \\
\text { Yantén }\end{array}$ & $\begin{array}{l}0.04 \\
0.04 \\
0.04 \\
0.12\end{array}$ & $\begin{array}{l}0.00 \\
0.00 \\
0.00 \\
0.00\end{array}$ & $\begin{array}{l}0.04 \\
0.04 \\
0.04 \\
0.12\end{array}$ & $\begin{array}{l}\text { me } \\
\text { me } \\
\text { me } \\
\text { me }\end{array}$ & $\begin{array}{l}E \\
E \\
E \\
P\end{array}$ \\
\hline Poaceae & Oryza sativa $\mathrm{L}$. & $\begin{array}{l}\text { Arroz, Arroz chino, Arroz } \\
3 \text { months, Arroz achica } 8, \\
\text { Arroz enano, Arroz } \\
\text { fortuno, Arroz uringa }\end{array}$ & 1.46 & 0.46 & 1.92 & fo & $\mathrm{P}$ \\
\hline & $\begin{array}{l}\text { Gynerium sagittatum P.Beauv. } \\
\text { Saccharum officinarum L. } \\
\text { Cymbopogon citratus Stapf } \\
\text { Zea mays L. } \\
\text { (LC) Agrostis perennans (Walter) Tuck. }\end{array}$ & $\begin{array}{l}\text { Cana brava } \\
\text { Canã } \\
\text { Limoncillo } \\
\text { Maiz } \\
\text { Paja }\end{array}$ & $\begin{array}{l}0.04 \\
0.38 \\
0.19 \\
0.35 \\
0.08\end{array}$ & $\begin{array}{l}0.00 \\
0.00 \\
0.00 \\
0.00 \\
0.00\end{array}$ & $\begin{array}{l}0.04 \\
0.38 \\
0.19 \\
0.35 \\
0.08\end{array}$ & $\begin{array}{l}\text { tc } \\
\text { fo, } \mathrm{m} / \mathrm{r} \text {, out } \\
\text { fo, me } \\
\text { fo, } \mathrm{me}, \mathrm{m} / \mathrm{r} \\
\mathrm{m} / \mathrm{r}\end{array}$ & $\begin{array}{l}\mathrm{E} \\
\mathrm{P} \\
\mathrm{P} \\
\mathrm{P} \\
\mathrm{E}\end{array}$ \\
\hline Portulacaceae & Talinum paniculatum (Jacq.) Gaertn. & Verdolaga & 0.04 & 0.00 & 0.04 & me & $\mathrm{E}$ \\
\hline Prrmulacede & (LC) Stylogyne turbacensis (Mez) & Calabonga, calabomba & 0.04 & 0.00 & 0.04 & $\mathrm{~m} / \mathrm{r}$ & $\mathrm{E}$ \\
\hline Rhizophoraceae & (LC) Rhizophora mangle $\mathrm{L}$. & Mangle & 0.08 & 0.00 & 0.08 & cs & $\mathrm{E}$ \\
\hline & $\begin{array}{l}\text { (LC) Borojoa patinoi Cuantec. } \\
\text { Morinda citrifolia } \mathrm{L} \text {. } \\
\text { (LC) Gonzalagunia ovatifolia B.L.Rob. } \\
\text { Psychotria poeppigiana Müll.Arg. }\end{array}$ & $\begin{array}{l}\text { BoroJó } \\
\text { Noni } \\
\text { Palito x } \\
\text { Sombrerito del diablo }\end{array}$ & $\begin{array}{l}0.15 \\
0.04 \\
0.08 \\
0.08\end{array}$ & $\begin{array}{l}0.04 \\
0.00 \\
0.00 \\
0.00\end{array}$ & $\begin{array}{l}0.19 \\
0.04 \\
0.08 \\
0.08\end{array}$ & $\begin{array}{l}\text { fo, me } \\
\text { me } \\
\text { me } \\
\text { me, } \mathrm{m} / \mathrm{r}\end{array}$ & $\begin{array}{l}P \\
P \\
E \\
E\end{array}$ \\
\hline Sapotaceae & $\begin{array}{l}\text { (LC) Pouteria caimito Radlk. } \\
\text { Manilkara zapota (L.) P.Royen }\end{array}$ & $\begin{array}{l}\text { Caimito } \\
\text { Níspero }\end{array}$ & $\begin{array}{l}0.08 \\
0.08\end{array}$ & $\begin{array}{l}0.00 \\
0.04\end{array}$ & $\begin{array}{l}0.08 \\
0.12\end{array}$ & $\begin{array}{l}\text { cs, fo } \\
\text { tc }\end{array}$ & $\begin{array}{l}\mathrm{E} \\
\mathrm{E}\end{array}$ \\
\hline Simaroubaceae & $\begin{array}{l}\text { (LC) Simarouba amara Aubl. } \\
\text { Simaba cedron Planch. }\end{array}$ & $\begin{array}{l}\text { Aliso } \\
\text { Cedrón }\end{array}$ & $\begin{array}{l}0.08 \\
0.31\end{array}$ & $\begin{array}{l}0.00 \\
0.04\end{array}$ & $\begin{array}{l}0.08 \\
0.35\end{array}$ & $\begin{array}{l}\text { fo, out } \\
\text { tc, me, } \mathrm{m} / \mathrm{r}\end{array}$ & $\begin{array}{l}\mathrm{E} \\
\mathrm{E}\end{array}$ \\
\hline Solanaceae & $\begin{array}{l}\text { (LC) Capsicum annuum } \mathbf{L} \text {. } \\
\text { Solanum incomptum Bitter } \\
\text { Solanum lycopersicum L. }\end{array}$ & $\begin{array}{l}\text { Aji dulce } \\
\text { Sauco } \\
\text { Tomate }\end{array}$ & $\begin{array}{l}0.08 \\
0.50 \\
0.04\end{array}$ & $\begin{array}{l}0.00 \\
0.00 \\
0.00\end{array}$ & $\begin{array}{l}0.08 \\
0.50 \\
0.04\end{array}$ & $\begin{array}{l}\text { fo, } \mathrm{m} / \mathrm{r} \\
\mathrm{me}, \mathrm{m} / \mathrm{r} \\
\text { fo }\end{array}$ & $\begin{array}{l}\mathrm{P} \\
\mathrm{P} \\
\mathrm{P}\end{array}$ \\
\hline
\end{tabular}




\begin{tabular}{|c|c|c|c|c|c|c|c|}
\hline \multicolumn{8}{|l|}{ Urticaceae } \\
\hline & Urera caracasana (Jacq.) Gaudich. ex Griseb. & Pringamosa & 0.04 & 0.00 & 0.04 & me & $\mathrm{E}$ \\
\hline & (LC) Cecropia peltata Vell. & Yarumo & 0.08 & 0.00 & 0.08 & me, out & $\mathrm{E}$ \\
\hline \multicolumn{8}{|l|}{ Verbenaceae } \\
\hline & $\begin{array}{l}\text { Lippia alba (Mill.) N.E.Br. ex Britton \& } \\
\text { P.Wilson }\end{array}$ & Pronto alivio & 0.23 & 0.00 & 0.23 & me & $\mathrm{P}$ \\
\hline \multicolumn{8}{|l|}{ Xantorreaceae } \\
\hline & Aloe vera $\mathrm{L}$. & Sábila & 0.12 & 0.00 & 0.12 & me & $\mathrm{P}$ \\
\hline \multicolumn{3}{|l|}{ Zingiberaceae } & & 0.00 & 0.15 & me, $\mathrm{m} / \mathrm{r}$ & $\mathrm{E}$ \\
\hline & Zingiber officinale Roscoe & Jengibre, Ajengibre & 0.04 & 0.00 & 0.04 & me & $\mathrm{P}$ \\
\hline
\end{tabular}
Note: $U_{\mathrm{a}}$ : current use value. $\mathrm{UV}_{\mathrm{p}}$ : potential use value. $\mathrm{UV}_{\mathrm{t}}$ total use value. UC: use category (me: medicinal, fo: food, tc: technological, fu: fuel/firewood, cs: construction, m/r: mystic/religious, out: others). P: planted, E: extractivism. Species in bold are in the IUCN Red List (LC: Least Concern, DD: Data Deficient, NT: Near Threatened, VU: Vulnerable)

Table 2. Ranking of the plant species considered most important by the Jovi community (Colombian Pacific) based on the UV = use value (UV total, UV current, UV potential). Species obtained by extractivism are marked with *. Species in bold are in the IUCN Red List

\begin{tabular}{|c|c|c|c|c|}
\hline Species & $\begin{array}{c}\begin{array}{c}\text { Popular } \\
\text { name }\end{array} \\
\end{array}$ & $\begin{array}{c}\mathrm{UV} \\
\text { total }\end{array}$ & $\begin{array}{c}\text { UV } \\
\text { current }\end{array}$ & $\begin{array}{c}\mathrm{UV} \\
\text { potential }\end{array}$ \\
\hline Musa x paradisíaca & Plátano & $2^{\circ}$ & $2^{\circ}$ & \\
\hline Oryza sativa & Arroz & $1^{\circ}$ & $1^{\mathrm{o}}$ & $1^{\circ}$ \\
\hline Dipteryx odorata & Choibá* & $3^{\circ}$ & $5^{\circ}$ & $2^{\circ}$ \\
\hline Cocos nucifera & Coco & $4^{\circ}$ & $3^{\circ}$ & \\
\hline Bactris gasipaes & Chontaduro & $5^{\circ}$ & & $2^{\circ}$ \\
\hline Solanum incomptum & Sauco & $5^{\circ}$ & $4^{\circ}$ & - \\
\hline Gliricidia sepium & Matarratón & - & $5^{\circ}$ & \\
\hline Anacardium excelsum & Espavé* & _ & - & $3^{\circ}$ \\
\hline Passiflora vitifolia & Granadillo* & - & - & $4^{\circ}$ \\
\hline Nectandra acutifolia & Jigua negro* & & & $5^{\circ}$ \\
\hline
\end{tabular}

\section{Discussion}

\section{Plants used by the Jovi community}

The Jovi community uses a wide variety of plants, with a similar number of cultivated species and species obtained by extractivism. However, the cultivated species showed the highest use values in the community. The plants with the highest values of total and current use were cultivated plants, i.e. Oryza sativa (rice) (1.92), and Musa x paradisiaca (plantano) (0.81). These species present exclusive food use and are cultivated by the community, which highlights their importance. The high use values of these two species were also reported in other AfroColombian communities in the municipality of Nuquí (Zuluaga and Ramírez 2015). Both, Musa x paradisiaca and Oryza sativa constitute the basis of the diet of the Afro-descendant population of Nuqui, as well as in most of Colombia (Morales and Caetano 2013). Their consumptions are present in the three daily meals, being used with great versatility of preparation and frequency in the cuisine of these communities (Zuluaga and Ramírez 2015; Alonso et al. 2014). The highest use values in the food category were also observed in Colombia (Morales and Caetano 2013).

The cultivation of Oryza sativa (rice), Musa $x$ paradisiaca (plantano), and Dipteryx odorata (choibá) usually does not exceed one hectare, and these species are intercropped. The first two species are well adapted to the soil and climate of the Colombian Pacific. The agricultural systems of the communities are based on crop rotation, cultivating on average three different species, lately inserting timbers to meet the economic demand, but with banano (banana) representing the main cultivated species (Zuluaga and Ramírez 2015). Although the habit of planting Oryza sativa (rice) is common in the community, this species showed a high potential use value (0.46), warning about possible losses in the use of this species in the community due to cultural changes (traditional production system) and planting management issues, such as the lack of knowledge about pest control, climate changes, land infertility, and decrease in the availability of native seeds (Ordõnez et al. 2018). In the case of the Jovi community, the losses are related to some specific varieties, such as 'fortune' and 'uringa', and leave to losses of traditional seeds.

Bactris gasipaes, Dipteryx odorata, and Anacardium excelsum also showed high potential use-value, corroborating the perception of the community on the use losses of species reported in the study conducted by Ordõnez et al. (2018). The use losses of plant species may be related to social, economic, and cultural changes faced by the Afro-Colombian communities of Nuqui. A study conducted in the same Department as this study showed that the propensity for the disappearance of ethnobotanical knowledge among young people who emigrate to the urban zone or dedicate to fishing activities resulted in a diluted need for the use of plant species, resulting in low usevalues due to the change of habits and culture (Galeano 2000). In Jovi, changes such as the migration of young people to the city in search of study and work and the increasingly growing work of women in the hotels, not dedicating much time to home-grown vegetable gardens and traditional culinary activities can be important factors that influence ethnobotanical knowledge. Similarly, the men look for economic ascension with other alternatives, such as timber cultivation or tourism (Zuluaga and Ramírez 2015; Ordõnez et al. 2018).

In general, the species showed low total use-value, indicating a restricted and limited use within the community (only one species showed total use value above 1.00 , and 106 species were allocated in the lowest 
classification interval ( 0.0 to 0.2$)$. This result can be further justified by the possible loss of interest in traditional knowledge by part of the community or due to the external influences to which these groups are subject (Crepaldi and Peixoto 2010; Vásquez 2013; Ordõnez et al. 2018), which was also reported in other Afro-descendant communities of South Africa (Lisboa 2017). It is important to highlight that the potential and current value indices focused on the specific use (of each use citation) of the species (Lucena et al. 2012). It means that we register the loss of some specific use of the species and it indicates a loss of the traditional knowledge, that can occur by cultural changes in the community or, instead, in changes and impact in the local biodiversity.

When analyzing the distribution of species by usecategory, it is possible to find similar results than other traditional populations, in which the highest numbers of cited species were indicated for medicinal use, followed by food purposes (Crepaldi and Peixoto 2010; Gomes and Bandeira 2012; Maroyi 2017). In the medicinal category, the families of Lamiaceae, Malvaceae, and Asteraceae were the most commonly used. Similar results were reported in studies conducted in other communities located in rainforest areas, such as in the Atlantic Forest region (Lisboa 2017), in other communities of the Colombian Pacific (Zuluaga and Ramírez 2015), and in the region of Angola, which, although featuring drought seasons in some periods of the year, has a prevailing humid tropical climate (Göhre et al. 2016). It should be highlighted that the Jovi community is geographically isolated, and access is only possible via boat, which complicates access to medical care. In this scenario, medicinal plants can have great importance in treating infirmities, especially milder ones. The distance between the community and urban areas or the means upon which this access depends seems to be determining factors to list the highest use values and number of cited species in the medicinal and food categories. Studies with hard-access Afro-descendant communities showed similar results, i.e. the more distant the region, the greater the dependence of the community on plant species (Göhre et al. 2016; Lautenschläger et al. 2018).

Other factors that can justify the ranking observed for the use-categories are the home-grown vegetable gardens and the subsistence agriculture activity since $53.41 \%$ of the species cited for the medicinal category and $79.63 \%$ of the species used for food purposes are traditionally cultivated by the community. In traditional communities, home gardens evidence the high relevance of the cultivation of these two categories and their importance for the nutritional establishment and treatment of infirmities (Silva et al. 2017). The maintenance of these cultivations goes beyond the food and nutrition security of the communities, fostering the local ecological and economic sustainability (Kahane et al. 2013).

The third use category identified by the community is the technological one, customarily used for manufacturing boats and tools for the community, without commercial purposes. The Arecaceae family is the most important in this category, obtained $100 \%$ by extractivism, although
Cocos nucifera is also cultivated by the community for food and medicinal purposes. The intense use of plant species for technological purposes was also observed in an isolated community in West Africa, highlighting the use of woody species (Ahoyo et al. 2018).

The use category for construction purposes highlights the use of plant species for house construction, with the highest number of cited species belonging to the families of Fabaceae and Lauraceae. The species cited for this purpose are obtained by extractivism $(84.62 \%)$, and planted by the community $(15.38 \%)$. In an Afro-descendant community close to the study area, in the Chocó coast, the use of plant species for construction purposes also relied on selective extractions from the forest for house construction, with the Lauraceae family being the most used (Galeano 2000). The study conducted by Galeano (2000) showed that most of the useful plants in the studied rainforest region were used for construction, technology, and firewood purposes. In Jovi, the least cited categories referred to the use of the species for fuel, whose employment occurs mainly as firewood for cooking, and in the "others" category, whose predominant use would be for household ornamentation, tourism, and recreation. Although there is a differentiation in the primary use categories between the present study and the one by Galeano (2000), a similarity is observed when both communities use the species for subsistence purposes, highlighting the importance of ethnobotanical investigation studies as they allow demonstrating that even close populations may present specificities, as well as demonstrate the temporal change that the communities can undergo.

Mystic/religious category is also a relevant category to the Jovi Community, especially in the cultural practice of "warding off the evil eye," focusing on the families of Poaceae, Piperaceae, and Solanaceae. The interviewees usually show a relation between the medicinal use and this category since it is common in Afro-descendant communities to use plant species for spiritual healing ceremonies to treat physical symptoms, such as fevers (Philander 2011).

Regarding the versatility of uses, the species of Clidemia septuplinervia (construction, technological, food, and medicinal purposes) and Saccharum officinarum (food, medicinal, mystic/ religious, and others) are the only ones that meet four use categories. In a region of Bolivia, four use-categories were also reported for Saccharum officinarum, although only the medicinal and food uses coincide (Zambrana et al. 2017). In another study conducted in the same Department as this study, only the food use was verified for this species (Zuluaga and Ramírez 2015). Saccharum officinarum was also highlighted in an Afro-descendant community of northeastern Brazil (Silva et al. 2017), where it is used as food and for treating infirmities.

Several studies suggest that the handling and knowledge of plants for medicinal and food purposes are a female domain (Cunha and Bortolotto 2011; Silva et al. 2012; De Carvalho et al. 2013; Silva et al. 2015; Göhre et al. 2016; Lisboa 2017); in this study, most interviewees were male $(67 \%)$, but no significant difference was verified 
regarding knowledge between men and women. Despite a difference of activities was related between men and women in the Jovi community, the men are responsible for collecting useful species when these are located in hardaccess areas, within the woods, or in distant places (Almeida et al. 2012; Silva 2012), it seems do not affect the ethnobotanical knowledge. A similar result was described in a community in Angola, where the interviewees reported the existence of work division between men and women, but with no significant difference (Lautenschläger et al. 2018).

Traditional knowledge among women in the Jovi region may be threatened by the economic and social dynamics, which is changing the roles of women in the community as they no longer have time to dedicate to the harvest since this activity competes directly with the services they provide in the surrounding hotel industry; also, younger women prefer to dedicate themselves to studying out of Jovi (Ordõnez et al. 2018). Another fact that should be considered regarding the knowledge between genders is that the local experts interviewed worked with agriculture $(50 \%)$, fishing $(27 \%)$, and tourism $(23 \%)$, with the first two activities being culturally performed by men, possibly resulting in a higher number of interviewees of this gender. The difference of activities can also result in specific knowledge, restricting individuals to certain species with different purposes, and contributing to low use-values. However, we believe that the interviewees are people with great knowledge, identified by the community as experts in the use of plant resources.

\section{Use and conservation}

The use of biodiversity for the most diverse purposes must be carefully observed and aiming to assess the impact that it can cause on the maintenance of the species populations. Jovi is a community located in a hot-spot region and information regarding the use of the species and its degree of threat should be the basis for management studies and the sustainable use of the species. In hot-spot regions, large numbers of species that require conservation priorities usually reflect high rates of extinction (Joppa et al. 2011). In this context, we highlight some species used by the Jovi community but also registered on the IUCN.

The use of two species in the Jovi community should be considered with extreme attention because they are in the most endangered categories of the IUCN, i.e. Minquartia guianensis and Pelliciera rhizophorae. The sustainable management of M. guianensis (Nebel 2001) is important due to the high extraction pressure and the depletion of natural populations in many regions, mainly in Brazil and Colombia (IUCN 2021). The Jovi community uses $M$. guianensis for construction purposes, and this species is recognized because of the hardness, durability, and resistance to pests of its wood (Oregon et al. 2018). This kind of use can hardly impact the species population because it reflects the death of the plant. The demand for local and commercial supply requires practices that lead to the increase of the density of trees as well as their growth rates (Nebel 2001). We emphasize the importance of the management process for $M$. guianensis, as the species is already recognized with potential use in the Jovi community, indicating a decrease in use (despite it is unknown whether it is due to a decrease of the population or for another reason).

The vulnerability of Pelliciera rhizophorae, indicated by the IUCN Red List, stems from the limited geographical distribution, as it has small and dispersed populations and is largely linked to the impacts generated by anthropic factors (Blanco-Libreros et al. 2015). Because it is a mangrove species, its distribution is suffering reduction and anthropic pressure in coastal regions (Blanco-Libreros et al. 2015; Duke 2020). Isolated populations and the low gene flow associated with anthropic pressures indicate that $P$. rhizophorae should be revised from Vulnerable to a higher threatened level on the IUCN List (Duke 2020). Considering that Jovi is located in a coastal and estuary region, uses $P$. rhizophorae and $M$. guianensis for timber purposes, and obtained both species by extractivism, it is necessary to develop a management plan specifically focused on the use of these more endangered species.

Gliricidia sepium (the 5th highest current use value in the Jovi community) and Nectandra acutifolia (the 5th highest potential use-value) are classified in the Least Concern category on the IUCN Red List, and no immediate threat to its genetic resources has been identified. Although medicinal plants, which occur in endemic habitats considered hot-spots, deserves attention regarding the priority of conservation and the intensity of its use (Dapar et al. 2020), in the case of G. sepium, we understand that the cultivating practice helps to reduce pressure on natural populations and can be a good strategy to reconcile traditional use with conservation practices. On the other hand, N. acutifolia is a species obtained by extraction and its use is focused on timber purposes, such as construction and technology in the Jovi community. It is also cited as medicinal in a community in Ecuador (Ballesteros et al. 2016). The use of $N$. acutifolia in the Jovi community makes the species more suitable to the ecological risk and deserves attention also in conservation studies. IUCN points out that deforestation and the conversion of natural habitats of this species to promote agricultural activities are potential threats, mainly in Colombia, Bolivia, Ecuador, and Peru (IUCN 2021).

One last species that is important to be highlighted in this discussion is Dipteryx odorata, which is the second place in the ranking of the potential use value and the 5 th in the ranking of the current use value. It is used for technological and construction uses, which, in general, cause the death of the plant. This species is indicated as Data Deficient on the IUCN Red List, so it is not possible to know the status or condition for maintaining the populations of this species. The impact on choibá ( $D$. odorata) has already been registered due to the growing exploitation for timber purposes for construction and technology (Rêgo et al. 2017; Oliveira 2020). Other factors that can justify the vulnerability of over-exploitation are biological characteristics such as reproductive capacity, needs for light, low dispersion rate, and high mortality rate (Herrero-Jauregui et al. 2011). Considering the type and importance of the use and the little knowledge about the 
populations of $D$. odorata, it is essential more studies focused on the ecology and development of the species in the region.

In conclusion, the Jovi community has a close relationship with plant resources, demonstrating great knowledge and versatility in the use of plant species. The ethnobotanical survey allowed verifying that approximately $57.23 \%$ of the species were attributed to medicinal purposes; for a community that is geographically isolated from traditional medical centers, these species have crucial importance in the treatment of infirmities and local health maintenance. A change in the food traditions is also verified, as this category showed the highest potential use values for the species cultivated by the community, such as Oryza sativa and Bactris gasipaes, indicating cultural changes.

The traditional use of the plants is important in the cultural perspective to the community; however, the community should also be included in a co-management plan focus on sustainable use, mainly considering that it is a hot-spot region. In this study, we identified threatened species used by extractivism and as a timber resource (such as Minquartia guianensis and Pelliciera rhizophorae). Even other forms of uses (as observed to Gliricidia sepium) and species that are Data Deficient, such as Dipteryx odorata, deserve more attention in the management plan. Some of these species are in the ranking of the five most important species to the community. The local government should include the community in this discussion, recognized their uses, and provide a wide discussion about sustainable use.

\section{REFERENCES}

Ahoyo CC, Houehanou TD, Yaoitcha AS, Prinz K, Assogbadjo AE, Adjahossou CS, Hellwing F, Houinato MR. 2018. A quantitative ethnobotanical approach toward biodiversity conservation of useful woody species in Wari-Maro forest reserve (Benin, West Africa). Environ Dev Sustain 20 (5): 2301-2320. DOI: 10.1007/s10668-0179990-0.

Albuquerque UP, Ramos MA, Lucena RFP, Alencar NL. 2014. Methods and Techniques Used to Collect Ethnobiological Data. In: Albuquerque U, Cunha LC, Lucena RFP, Alves R (eds) Methods and Techniques in Ethnobiology and Ethnoecology. Springer Protocols Handbooks. Humana Press, New York, NY. DOI: 10.1007/978-14614-8636-7_2.

Almeida CFCBR, Ramos MA, Silva RRV, Melo JG, Medeiros MFT, Araújo TAS, Almeida ALS, Amorim ELC, Alves RRN, Albuquerque UP. 2012. Intracultural variation in the knowledge of medicinal plants in an urban-rural community in the Atlantic Forest from Northeastern Brazil. Evid-Based Compl Altern Med 2012: 679373. DOI: $10.1155 / 2012 / 679373$.

Alonso C, Villarreal AMA, Cortés EA, Arévalo PA, Baptiste MP, Bello LC, Carrascal RAC. 2014. Biodiversidad 2014. Estado y tendencias de la biodiversidad continental de Colombia. Instituto Alexander von Humboldt. Bogotá D.C., Colombia.

Ballesteros JL, Bracco F, Cerna M, Vita FP, Vidari G. 2016 Ethnobotanical research at the Kutukú Scientific Station, MoronaSantiago, Ecuador. BioMed Res Intl 2016: 9105746. DOI:10.1155/2016/9105746.

Bernard HR. 2017. Research Methods in Anthropology: Qualitative and Quantitative Approaches. $6^{\text {th }}$ ed. Rowman e Littlefield, London.

Blanco-Libreros JF, Estrada-Urrea EA, Pérez-Montalvo RJ, TabordaMarín A, Álvarez-León R. 2015. Influencia antrópica en el paisaje de las poblaciones de Pelliciera rhizophorae (Ericales: Tetrameristaceae) más sureñas del Caribe (Turbo, Colombia). Rev Biol Trop 63 (1): 927-942. DOI: 10.15517/rbt.v63i4.16845.

Crepaldi MOS, Peixoto AL. 2010. Use and knowledge of plants by "Quilombolas" as subsidies for conservation efforts in an area of Atlantic Forest in Espírito Santo State, Brazil. Biodivers Conserv 19 (1): 37-60. DOI: 10.1007/s10531-009-9700-9.

Cunha SAD, Bortolotto IM. 2011. Etnobotânica de plantas medicinais no assentamento Monjolinho, município de Anastácio, Mato Grosso do Sul, Brasil. Acta Bot Bras 25 (3): 685-698. DOI: 10.1590/S010233062011000300022.

Dapar MLG, Alejandro GJD. 2020. Ethnobotanical studies on indigenous communities in the Philippines: current status, challenges, recommendations and future perspectives. J Compl Med Res 11 (1): 432-446. DOI: 10.5455 / jcmr.2020.11.01.51.

De Carvalho JSB, Martins JDL, Mendonça MDCS, de Lima LD. 2013. Uso popular das plantas medicinais na comunidade da Várzea, Garanhuns-PE. Rev Biol Ciênc Terra 13 (2): 58-65.

Diegues AC. 2019. Conhecimentos, práticas tradicionais e a etnoconservação da natureza. Desenvolvimento e Meio Ambiente 50: 116-126. DOI: 10.5380/dma.v50i0.66617.

Duke NC. 2020. A systematic revision of the vulnerable mangrove genus Pelliciera (Tetrameristaceae) in equatorial America. BlumeaBiodivers Evol Biogeogr Plants 65: 107-120. DOI: 10.3767/blumea.2020.65.02.04.

Galeano G. 2000. Forest use at the Pacific Coast of Chocó, Colômbia: a quantitative approach. Bot Econ 54 (3): 358-376. DOI: 10.1007/BF02864787.

Göhre A, Toto-Nienguesse AB, Futuro M, Neinhuis C, Lautenschläger T. 2016. Plants from disturbed savannah vegetation and their usage by Bakongo tribes in Uíge, Northern Angola. J Ethnobiol Ethnomed 12 (1): 1-28. DOI: 10.1186/s13002-016-0116-9.

Gomes TB, Bandeira FDF. 2012. The use and diversity of medicinal plants in a quilombola community in Raso da Catarina, Bahia. Acta Bot Bras 26 (4): 796-809. DOI: 10.1590/S0102-33062012000400009.

Harisha RP, Padmavathy S, Nagaraja BC. 2015. Traditional Ecological Knowledge (TEK) and its importance in South India. Appl Ecol Environ 14: 311-326. DOI: 10.15666/aeer/1401_311326.

Herrero-Jáuregui C, García-Fernández C, Sist PL, Casado MA. 2011. Recruitment dynamics of two low-density neotropical multiple-use tree species. Plant Ecol 212 (9): 1501-1512. DOI: 10.1007/s11258011-9924-0.

Braham WK, Blandón MM. 2013. Plan Estratégico de la Macrocuenca del Pacífico. IIAP - Instituto de Investigaciones Ambientales del Pacifico. https://www.minambiente.gov.co/index.php/gestionintegral-del-recurso-hidrico/planificacion-de-cuencas-

hidrograficas/macrocuencas/avances-en-la-formulacion-de-losplanes-estrategicos

Joppa LN, Roberts DL, Myers N, Pimm SL. 2011. Biodiversity hotspots house most undiscovered plant species. Proc Nat Acad Sci 108 (32): 13171-13176. DOI: 10.1073/pnas.1109389108

Kahane R, Hodgkin T, Jaenicke H, Hoogendoorn C, Hermann H, Hughes JDA, Looney N. 2013. Agrobiodiversity for food security, health and income. Agron Sustain Dev 33 (4): 671-693. DOI: 10.1007/s13593013-0147-8.

Kakoty S. 2018. Ecology, sustainability and traditional wisdom. J Cleaner Prod 172: 3215-3224. DOI: 10.1016/j.jclepro.2017.11.036

Lautenschläger T, Monizi M, Pedro P, Mandombe JL, Bránquima MF, Heinze C, Neinhuis C. 2018. First large-scale ethnobotanical survey in the province of Uíge, northern Angola. J Ethnobiol Ethnomed 14 (1): 1-73. DOI: 10.1186/s13002-018-0238-3.

Lisboa MS. 2017. Estudo etnobotânico em comunidade quilombola Salamina/Putumujú em Maragogipe, Bahia. Rev Fitos 11: 48-61. DOI: $10.5935 / 2446-4775.20170006$

Lucena RFP, Medeiros PM, Araújo EF, Alves AGC Albuquerque UP. 2012. The ecological apparency hypothesis and the importance of useful plants in rural communities from Northeastern Brazil: An assessment based on use value. J Environ Manag 96: 106-115. DOI: 10.1016/j.jenvman.2011.09.001

Maroyi A. 2017. Diversity of use and local knowledge of wild and cultivated plants in the Eastern Cape province, South Africa. J Ethnobiol Ethnomed 13 (1): 1-16. DOI: 10.1186/s13002-017-0173-8.

MEA (Millennium Ecosystem Assessment). Ecosystems and human wellbeing: wetlands and water. 2005. http://hdl.handle.net/10919/65899.

Mittermeier RA, Gil PR, Hoffman M, Pilgrim J, Brooks T, Mittermeier C, Saligmann AP. 2004. Hotspots Revisited: Earth's Biologically 
Richest and Most Endangered Terrestrial Ecoregions. Cemex, Mexico City.

Morales MMB, Caetano CM. 2013. Inventory and valuation of flora used by the village Santa Teresa, Palmira (Valle del Cauca)/ Inventario y valoración de la flora utilizada por la vereda Santa Teresa, Palmira (Valle del Cauca)/Inventario e avaliacao da flora utilizada pela vila de Santa Teresa, Palmira (Valle del Cauca). Revista de Investigacion Agraria y Ambiental 4: 89-100. DOI: 10.22490/21456453.985.

Myers N, Mittermeier RA, Mittermeier CG, Fonseca GA, Kent J. 2000. Biodiversity hotspots for conservation priorities. Nature 403 (6772): 853-858. DOI: 10.1038/35002501.

Nebel G. 2001. Minquartia guianensis Aubl: use, ecology and management in forestry and agroforestry. For Ecol Manag 150 (1-2) 115-124. DOI: 10.1016/S0378-1127(00)00685-X.

Oliveira PC. 2020. Traditional knowledge of forest medicinal plants of munduruku indigenous people-ipaupixuna. Eur J Med Plants 31: 2035. DOI: $10.9734 /$ ejmp/2020/v31i1330309.

Ordóñez LL, Cruz DD, Andrade MO. 2018. Ecosystem services and use of Afro-descendant land in the Colombian North Pacific: Transformations in the traditional production system. Land Use Policy 75: 631-641. DOI: 10.1016/j.landusepol.2018.01.043.

Philander LA. 2011. An ethnobotany of Western Cape Rasta bush medicine. J Ethnopharmacol 138 (2): 578-594. DOI: 10.1016/j.jep.2011.10.004.

Rêgo LJS, da Silva ML, da Silva LF, Gama JRV, Reis LP. 2017. Comercialização da amêndoa de cumaru nos municípios de Santarém e Alenquer, leste da Amazônia. Rev Administração e Negócios da Amazônia 8 (3): 338-361. DOI: 10.18361/2176-8366/rara.v8n3p338361 .
Restrepo E. 2013. El giro a la biodiversidad en la imaginación del Pacífico colombiano. Rev Estud Pac Colomb 1 (1): 171-199.

Riscales, Consejo Comunitario General Los. 2005. Historia, Territorio y Cultura. Chicó, Colombia.

Rossato SC, Leitão-Filho HF, Begossi A. 1999. Ethnobotany of caiçaras of the Atlantic Forest coast (Brazil). Econ Bot 53 (4): 387-395. DOI: 10.1007/BF02866716.

RStudio Team. 2020. RStudio: Integrated Development for R. RStudio, PBC, Boston, MA. http://www.rstudio.com/.

Silva MDP, Marini FS, Melo RS. 2015. Levantamento de plantas medicinais cultivadas no município de Solânea, agreste paraibano: reconhecimento e valorização do saber tradicional. Rev Bras Plantas Med 17 (4): 881-890. DOI: 10.1590/1983-084X/14_112.

Silva NCB, Regis ACD, Esquibel MA, Santos JDES, Almeida MZ. 2012. Medicinal plants use in Barra II quilombola community - Bahia, Brazil. Boletín Latinoamericano y del Caribe de Plantas Medicinales y Aromáticas 11 (5): 435-453.

Silva PH, Oliveira YR, Abreu MC. 2017. Uma abordagem etnobotânica acerca das plantas úteis cultivadas em quintais em uma comunidade rural do semiárido piauiense, Nordeste do Brasil. J Environ Anal Prog 2 (2): 144-159. DOI: 10.24221/jeap.2.2.2017.1175.144-159.

Zambrana NYP, Bussmann RW, Hart RE, Huanca ALM, Soria GO, Vaca MO, Moreno BC. 2017. Traditional knowledge hiding in plain sighttwenty-first century ethnobotany of the Chácobo in Beni, Bolivia. J Ethnobiol Ethnomed 13 (1): 1-57. DOI: 10.1186/s13002-017-0179-2.

Zuluaga GP, Ramírez LA. 2015. Uso, manejo y conservación de la agrobiodiversidad por comunidades campesinas afrocolombianas en el municipio de Nuquí. Colomb Etnobiol 13: 5-18. 